\title{
Metal-organic framework (MOF) as a novel humidity control material for autonomous indoor moisture management
}

\author{
Menghao Qin*, Kan Zu, Pumin Hou \\ Department of Civl Engineering, Technical University of Denmark (DTU), Lyngby, Denmark
}

\begin{abstract}
ASHRAE recommends that the appropriate indoor relative humidity range for a healthy and comfortable indoor environment is between $40 \%$ and $65 \% \mathrm{RH}$. In order to meet the requirement, the vapour-compression air-conditioning system is the most commonly used method for dehumidification. However, this approach is energy-consuming. In this paper, a novel precise humidity control material (PHCM) based on Metal-Organic Frameworks (MOFs) is synthesized. This material has an S-shape isotherm, high porosity, and very high water vapor uptake of $1.62 \mathrm{~g} / \mathrm{g}$ at $80 \% \mathrm{RH}$. MOF-PHCM can autonomously control indoor relative humidity within the desired comfort range at room temperature. Hygrothermal properties of the new material are measured. Numerical simulations have been carried out to study the effect of MOF-PHCM on indoor hygrothermal conditions and building energy consumption in different climates. The results show that MOF-PHCM can effectively control indoor relative humidity fluctuations and reduce building energy consumption in most climates without any additional energy input. Peer-review under the responsibility of the organizing committee of the ICMB21.
\end{abstract}

Keywords: MOFs; Precise humidity control material; Indoor relative humidity; Building energy saving

\section{Introduction/Background}

Indoor relative humidity is an important parameter to determine indoor air quality, occupants' thermal comfort and building energy consumption. In order to maintain indoor comfort and to prevent microorganism growth, the American Society of Heating, Refrigerating and Air-Conditioning Engineers (ASHRAE) recommends that indoor relative humidity should be maintained between $40 \%$ and $65 \%$. In the conventional air-conditioning system, air is cooled and dehumidified simultaneously. The latent cooling load is removed by the refrigeration dehumidification process. Air is cooled to the dew point first, and then re-heated to the set-point for indoor environment, which results in wasting a lot of energy. Desiccants help the system to independently control both temperature and humidity and thus contribute to the reduction of the energy used [1]. Silica gels and zeolites are the most commonly used solid desiccants, but they are not very efficient for dehumidification owing to their high regeneration temperature, long cycling time and the fact that a larger part of their moisture sorption occurs outside the desired comfort relative humidity range (i.e. between $40 \%$ and $65 \% \mathrm{RH}$ ). Dehumidification by liquid sorbents uses less electrical energy than refrigeration, but the relevant technology has some inherent defects, e.g. the system is complex and bulky, and has corrosion problems.

The ideal materials for autonomous regulation of indoor humidity should meet the following criteria: (1) The material should have a S-shape isotherm and exhibit a steep uptake isotherm at a specific RH depending on the targeted application. For indoor thermal comfort, the adsorption isotherm must have a steep rise around $65 \% \mathrm{RH}$, and the desorption isotherm must have a steep decrease around 40\% RH (see Fig. 1); (2) High water vapor uptake within the operating vapour pressure window; (3) Low regeneration temperature and high reproducible cycling performance; (4) High hygrothermal stability, non-toxicity and noncorrosion. Solid porous material that meets all above four criteria is named as Precise Humidity Control Material (PHCM).

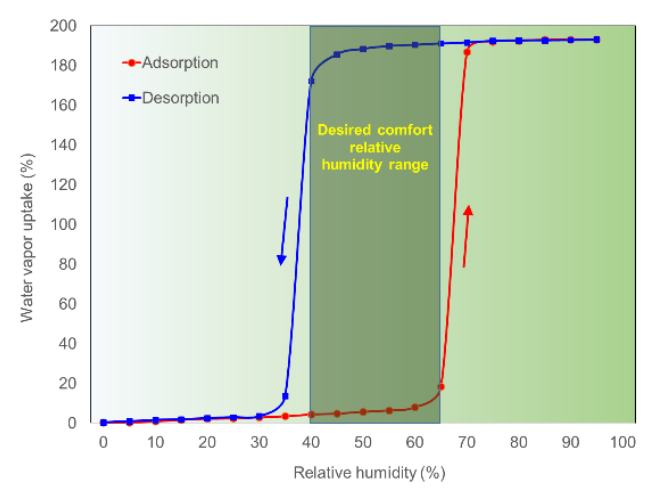

Figure 1. Idea water vapour isotherms of precise humidity control material

\footnotetext{
* Corresponding author. Tel.: +45 5036 5768, Email: menqin@byg.dtu.dk, Website: https://www.staff.dtu.dk/menqin/home
} 


\section{ICMB21}

Metal-organic frameworks (MOFs) are a new class of porous materials. Most metal-organic frameworks have very high surface areas and large adsorption capacity for gas. Due to their functional and structural tunability, metal-organic frameworks have become one of the most fascinating classes of materials for both scientists and engineers [2]. Recent studies show that metalorganic frameworks are also promising adsorbents for water vapour [1,3]. Some metal-organic frameworks, such as Al-fumarate, CAU-10-H, PIZOF-2, MIL-101, MIL-101-NH2, Y-shp-MOF5, and MIL-100(Fe) show good water vapor adsorption properties for humidity control [4], and have different operating pressure windows in a general range of 20\%-80\% RH. However, to the best of our knowledge, only very few of metal-organic frameworks can be used for autonomous moisture control for human comfort. In addition, most previous studies about metal-organic frameworks are from chemistry and physics, and mainly focus on the synthesis and characterization of novel composites. Detailed studies of the effect of metal-organic frameworks on built environment and building energy consumptions have not been reported.

This paper aims to first develop a new MOF-based PHCM that can realize the autonomous control of indoor moisture, and then study the performance of the new MOF-PHCM for the indoor moisture control in different climates.

\section{Materials and Characterization}

The preparation and characterization of the new MOF based PHCM were carried out at DTU Civil Engineering and DTU Chemistry. The scanning electronic microscope (SEM) is shown in Fig. 2; and the water vapor isotherms measured by a DVS instrument is shown in Fig. 3. The water vapor uptake of MOF-PHCM at $80 \% \mathrm{RH}$ is $1.62 \mathrm{~g} / \mathrm{g}$, which is much higher than most conventional sorbents and many MOFs [4]. The moisture buffer value (MBV) of the MOF-PHCM was measured according to the classic MBV test method proposed by NORDTEST [5]. The test results show that the MBV of MOF-PHCM is $20.50 \mathrm{~g} \cdot \mathrm{m}^{-2} \cdot \mathrm{RH}^{-1}$ in the experimental conditions, which is almost 45 times higher than that of laminated wood and 36 times higher than gypsum [4].

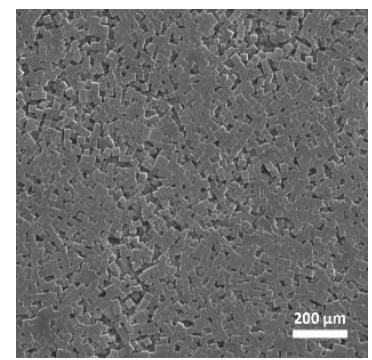

Figure 2. SEM image of MOF-PHCM crystals

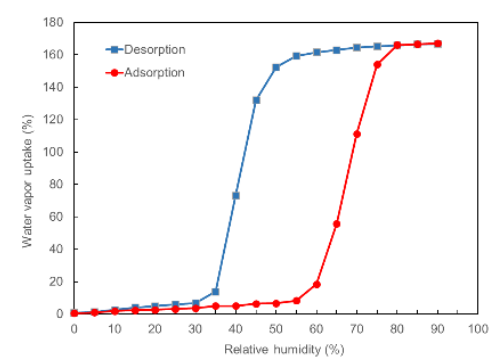

Figure 3. Water vapour sorption isotherms of MOF-PHCM at $23^{\circ} \mathrm{C}$.

\section{Simulation}

Both the water vapour sorption isotherms and the MBV test show that the MOF-PHCM has an extraordinary moisture buffering capacity, which makes it very promising for indoor passive moisture control. Numerical simulations using WUFI+ were carried out to study the effect of MOF-PHCM on indoor humidity condition and building energy consumptions. The energy saving potential of applying MOF-PHCM in built environment in different climates has been investigated. Five typical climates/cities worldwide are selected. They are: humid subtropical climate (e.g. Hong Kong), temperate climate (e.g. London), moderate Mediterranean climate (e.g. Madrid), semi-arid climate (e.g. Salt Lake City), and hot desert climate (e.g. Phoenix).

The simulation results show that the novel MOF-PHCM could be used for indoor humidity control in all climates. In Phoenix, Salt Lake City and Madrid climate, MOF-PHCM could autonomously control indoor RH within the comfort zone without any additional energy input. In London climate, a single MOF-PHCM wall panel could remove $52.6 \%$ of the total indoor latent load without additional energy input. If the material is regenerated by a proper regeneration system, for example, heating systems powered by renewable energy, enough MOF-PHCM could also realize the autonomous regulation of indoor RH in temperate climate. In Hong Kong climate, MOF-PHCM must be integrated with a proper regeneration system to realize the indoor humidity control. It is important to note that the amount of MOF-PHCM for moisture control in different climates should be calculated according to the indoor moisture load and outdoor weather condition.

\section{References}

[1] S. Cui, M. Qin et al. (2018). Metal-Organic Frameworks as advanced moisture sorbents for energy-efficient high temperature cooling, Nature, Scientific Reports, Vol. 8.

[2] H. Furukawa, K. Cordova, M. O'Keeffe, O. Yaghi (2013). The Chemistry and Applications of Metal-Organic Frameworks, Science.

[3] K. Zu, M. Qin, S. Cui (2020) Progress and potential of metal-organic frameworks (MOFs) as novel desiccants for built environment control: A review. Renewable \& Sustainable Energy Reviews, Vol. 133.

[4] X. Feng, M. Qin, S. Cui, C. Rode (2018). Metal-Organic Framework MIL-100(Fe) as a Novel Moisture Buffer Material for Energy-Efficient Indoor Humidity Control, Building and Environment, Vol. 145.

[5] M. Zhang, M. Qin, C. Rode, Z. Chen (2017). Moisture buffering phenomenon and its impact on building energy consumption, Applied Thermal Engineering, Vol.124 337-345. 